\title{
Clinical progression and metachronous paragangliomas in a large cohort of SDHD germline variant carriers
}

\author{
Berdine L Heesterman ${ }^{1} \cdot$ Lisa M H de Pont ${ }^{1} \cdot$ Andel GL van der Mey ${ }^{1}$. Jean-Pierre Bayley ${ }^{2}$ Eleonora PM Corssmit ${ }^{3}$. \\ Frederik J Hes $\mathbb{B}^{4} \cdot$ Berit M Verbist ${ }^{5}$. Peter Paul G van Benthem ${ }^{1}$. Jeroen C Jansen ${ }^{1}$
}

Received: 25 February 2017 / Revised: 28 January 2018 / Accepted: 1 February 2018 / Published online: 18 May 2018

(c) European Society of Human Genetics 2018

\begin{abstract}
Although it is well established that paternally transmitted germline variants in SDHD are associated with multifocal paragangliomas and lifelong follow-up is generally advised, the risk of metachronous lesions is presently unknown. In a large Dutch cohort of SDHD variant carriers, we studied the development of new paragangliomas, and the evolution of symptoms and cranial nerve impairment. Recurrent event analysis and the Kaplan-Meier product limit estimator were used to study the risk of new lesions. The relation between several predictors and development of new symptoms was assessed using logistic regression. Of the 222 SDHD variant carriers included, $65 \%$ presented with symptoms and $11 \%$ with cranial nerve dysfunction. Over a median period of 8 years, $42 \%$ reported new symptoms, and new cranial nerve impairment was observed in $11 \%$ of subjects. The estimated fraction of subjects that developed new HNPGL increased to 73\% (95\% CI: 52-85\%) after 22 years of follow-up. Males were more likely to develop new HNPGL compared to females (HR: 1.63, 95\% CI: 1.10-2.40), as were subjects that presented with symptoms, compared to subjects that were asymptomatic at baseline (HR: 1.61, 95\% CI: 1.01-2.55). In addition, the risk of new lesions decreased with number of HNPGL present at first diagnosis (HR: 0.68 and 95\% CI: 0.56-0.82). Carriers of a paternally inherited SDHD variant face a considerable risk for new HNPGL. In addition, nearly $50 \%$ of subjects reported new symptoms. However, new cranial nerve deficits were observed in only $11 \%$, which is less than reported in surgical series. These risks should be taken into account when considering treatment strategies and counseling.
\end{abstract}

Electronic supplementary material The online version of this article (https://doi.org/10.1038/s41431-018-0116-4) contains supplementary material, which is available to authorized users.

Berdine L Heesterman

B.L.Heesterman@outlook.com

1 Department of Otorhinolaryngology, Leiden University Medical Center, Leiden, The Netherlands

2 Department of Human Genetics, Leiden University Medical Center, Leiden, The Netherlands

3 Department of Endocrinology, Leiden University Medical Center, Leiden, The Netherlands

4 Department of Clinical Genetics, Leiden University Medical Center, Leiden, The Netherlands

5 Department of Radiology, Leiden University Medical Center, Leiden, The Netherlands

\section{Introduction}

Hereditary head and neck paragangliomas (HNPGL) are primarily associated with germline variants in the genes encoding subunits of succinate dehydrogenase (SDHA, SDHB, SDHC, and SDHD) or its assembly factor (SDHAF2). SDHD variants are the leading cause of hereditary head and neck paragangliomas in the Netherlands, and a high prevalence of two founder variants, c.274G $>\mathrm{T}$, p.(Asp92Tyr) and c.416T >C, p.(Leu139Pro), is observed in the Dutch population [1-3]. A remarkable parent-of-origin effect characterizes inheritance of SDHD-related paragangliomas (PGL). Carriers of a germline variant in this gene develop a phenotype almost exclusively upon paternal transmission. Although still unproven, the hypothesis that a second paternally imprinted gene, presumably located on $11 \mathrm{p} 15$, is involved in tumorigenesis seems the most plausible explanation to date. A requirement for complex mitotic recombination of the maternal $11 \mathrm{q}$ and paternal $11 \mathrm{p}$ region, followed by loss of the paternal $11 \mathrm{q}$ and maternal 
$11 \mathrm{p}$ region, explains the rare occurrence of maternally transmitted disease [4-7].

Numerous authors have studied genotype-phenotype correlations, and SDHD variants are typically associated with head and neck paragangliomas, multifocal disease, and a low malignancy rate. Germline variants in SDHD also predispose carriers to develop pheochromocytomas (PCC) and extra-adrenal sympathetic paragangliomas (sPGL) [812]. Patients may present with symptoms related to mass effect or occasionally with symptoms caused by excessive catecholamine secretion. In addition, HNPGLs are increasingly detected following screening by genetic testing and imaging in the context of hereditary disease [13].

Although a few authors have reported metachronous lesions in individual patients, the risk of developing new head and neck paragangliomas during follow-up has not been previously studied [14-18]. In addition, there are no large studies describing clinical progression in patients with untreated HNPGL.

With the aim to further optimize surveillance and counseling of both patients and their family members, we studied symptoms and cranial nerve dysfunction at initial presentation, clinical progression, and the development of new paragangliomas in a large Dutch cohort of SDHD variant carriers.

\section{Methods}

\section{Subjects}

The database of the Laboratory for Diagnostic Genome Analysis (LDGA) of the Leiden University Medical Center, a tertiary referral center for patients with PGL in the Netherlands, was used to identify SDHD variant carriers. Molecular genetic testing was performed as previously described (reference sequence: NT_033899.7 NM_003002.2) [19]. In addition, family members known at the Leiden University Medical Center with an obligate carrier status, which requires at least one diagnosed paraganglioma and a family member with a germline SDHD variant, were eligible for inclusion. Subjects diagnosed with PGL between January 1990 and October 2015 were included if, following first diagnosis, they underwent imaging in our institution at least once and visited the Department of Otorhinolaryngology, Endocrinology, or Surgery. Carriers of a paternally inherited SDHD variant, with no evidence of disease at initial surveillance, were included if at least one additional MRI or CT scan was available.

In accordance with the Dutch law, approval of the institutional ethics committee was not required because all data used were collected for routine patient care.

\section{Surveillance}

Magnetic resonance imaging (MRI) is generally used in our institution for the detection and follow-up of HNPGL (contrast-enhanced 3D time of flight MR angiography sequence has been used since the late 1990s). If there are contraindications for magnetic resonance imaging (e.g., implanted cardioverter-defibrillator or claustrophobia), computed tomography (CT) is used. Measurement of urinary catecholamines and their $O$-methylated metabolites, to detect hormonally active paragangliomas, was performed as described by Havekes et al. and was followed by MRI or CT scans of the thorax, abdomen, and pelvis in case of excessive catecholamine secretion $[18,20]$. If a sPGL or PCC was suspected, ${ }^{123}$ I metaiodobenzylguanidine (MIBG) scintigraphy was performed. Since 2002, biochemical screening has been performed at 2-year intervals and MR imaging at intervals of 1-2 years (every 5 years if no evidence of disease is found).

Occasionally, carotid body tumors were detected by head and neck ultrasonography and jugulotympanic tumors by CT imaging of the temporal bone. In light of the risk of multifocal disease associated with SDHD variants, crosssectional imaging of the head and neck region should be added to discover additional PGLs. With this in mind, the development of a new primary paraganglioma was defined as the detection of a tumor at least 1 year after initial diagnosis. Accordingly, tumors detected within the first year of follow-up were classified as present at baseline.

The starting point for follow-up was the first MRI or CT scan of the head and neck region of SDHD variant carriers without evidence of disease at initial surveillance. For the remaining subjects, the starting point was equivalent to the date of diagnosis of the first PGL. The time of most recent imaging of the head and neck region was considered the end of follow-up, as it was the most recent point at which new HNPGL could be detected. Relevant clinical data were retrieved from medical records, and comprised the period to the last PGL-related visit to the LUMC. Common PGLrelated signs and symptoms (e.g., neck swelling, hearing loss, tinnitus, dysphonia, palpitations, hypertension, and cranial nerve dysfunction) were routinely assessed.

\section{Statistics}

For statistical analysis, IBM SPSS Statistics version 23 (IBM Corp.: Armonk, NY, USA) and R version 3.2.5 were used. To assess the risk of developing new (metachronous) HNPGL, recurrent event analysis was used (more specifically a Prentice, Williams and Peterson Total Time model, an extension of Cox proportional hazards regression) [21, 22]. The proportional hazards assumption was checked using scaled Schoenfeld residuals. Age at the start of 

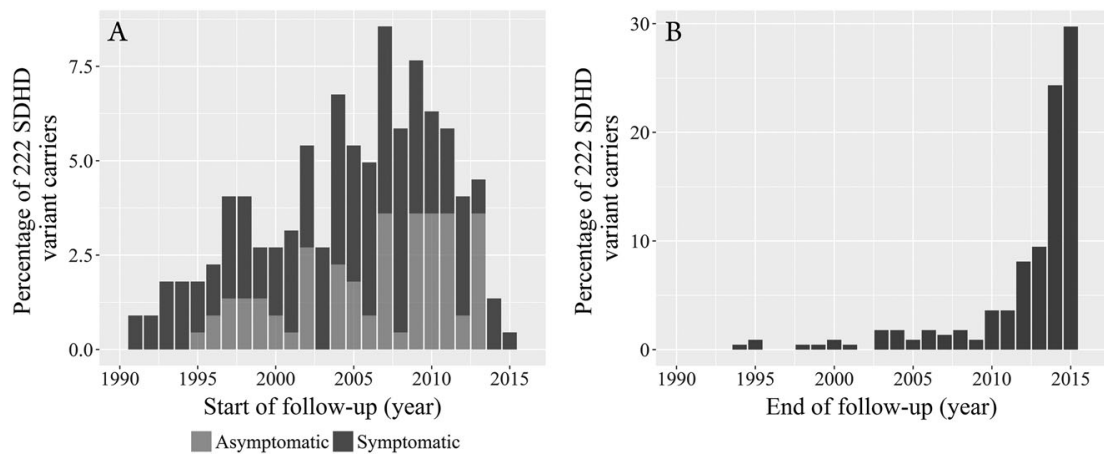

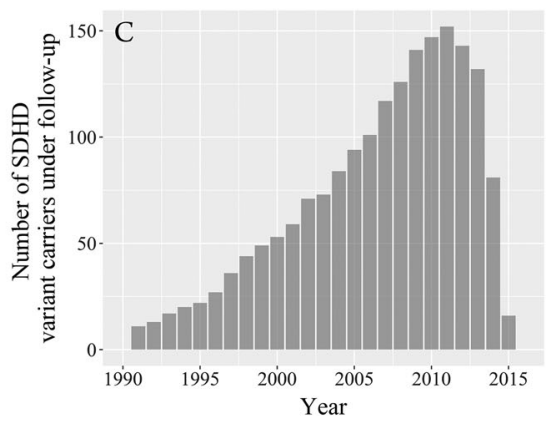

Fig. 1 The year subjects were included are depicted in Fig. 1a. In addition, the proportion of subjects that presented with symptoms versus the proportion of subjects that were asymptomatic at the start of

Table 1 Baseline characteristics

\begin{tabular}{lll}
\hline & Median/N & IQR/\% \\
\hline $\begin{array}{lll}\text { Gender } \\
\text { Male }\end{array}$ & 121 & $55 \%$ \\
$\quad$ Female & 101 & $45 \%$ \\
Mutation ${ }^{\mathrm{a}}$ & & \\
$\quad$ c.274G $>$ T p.(Asp92Tyr) & 177 & $80 \%$ \\
$\quad$ c.416T $>$ C p.(Leu139Pro) & 27 & $12 \%$ \\
Other & 18 & $8 \%$ \\
Age & & \\
$\quad$ Age at the start follow-up $(n=222)^{\mathrm{b}}$ & 39 & $29-49$ \\
$\quad$ Age at diagnosis $(n=213)^{\mathrm{b}}$ & 39 & $29-49$ \\
Symptomatic at baseline & 145 & $65 \%$ \\
Asymptomatic at baseline & 77 & $33 \%$ \\
Median no. of head and neck paragangliomas & 2 & $1-3$ \\
\hline
\end{tabular}

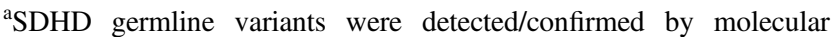
genetic testing (reference sequence: NT_033899.7 NM_003002.2) in 179 cases $(81 \%)$; the remaining subjects were obligate carriers. The other SDHD variants detected in the study population are listed in the supplementary information

${ }^{\mathrm{b}}$ The date of diagnosis was equal to the start of follow-up for 208 patients. Five subjects developed the first paraganglioma during follow-up (age at diagnosis $>$ age at the start of follow-up) and nine SDHD variant carriers remained disease free. For 27 patients, only the year of diagnosis was known, month and day were set to 1st January

follow-up, gender, whether a patient was symptomatic or asymptomatic at baseline, and the number of head and neck paragangliomas present at the start of follow-up were considered possible predictors. As imaging techniques have improved over time, we also included the year follow-up started as predictor. Although we intended to include several SDHD variants in our analysis, the high prevalence of the c.274G $>$ T, p.(Asp92Tyr) variant and the much lower number of subjects and observed events for other variants precluded reliable comparison. Only seven patients developed more than three new primary head and neck paragangliomas, therefore a data set limited to a maximum of follow-up is specified. The end of follow-up (year of most recent imaging) is depicted in Fig. $1 \mathrm{~b}$ and the total number of SDHD variant carriers under follow-up each year is provided in Fig. 1c

three events was used for recurrent event analysis [21]. The estimated fraction of subjects developing at least one new HNPGL and the median time to this first event was provided by the Kaplan-Meier product limit estimator. To illustrate the effect of binary predictors, Cox proportional hazards regression was used.

The relation between aforementioned predictors, as well as the number of new HNPGL, the development of new symptoms (attributable to paragangliomas located in the head and neck region), and cranial nerve paresis or paralysis was assessed with logistic regression. To correct for varying follow-up times, the duration of follow-up was included in the analysis. To compare the age at onset, defined as the age a patient retrospectively first experienced symptoms, of males versus females, an independent sample $t$-test was performed. If continuous data followed a Gaussian distribution, the mean and standard deviation are provided, if not, the median and interquartile range (IQR) are given unless stated otherwise. A $p$ value of less than 0.05 was considered statistically significant.

\section{Results}

\section{Patients}

A total of 222 Dutch SDHD variant carriers were included, $121(55 \%)$ of whom were male. One subject had developed HNPGL upon maternal transmission, whereas in the remaining 221 subjects the SDHD variant was transmitted via the paternal line. Figure 1 depicts the number of patients under follow-up from 1990 to 2016. The SDHD c.274G>T, p.(Asp92Tyr) variant was present in $80 \%$, and the SDHD c.416T $>$ C, p.(Leu139Pro) variant in $12 \%$ of subjects. The LOVD database (http://www.LOVD.nl/SDHD) identification numbers and SDHD variants present in the remaining subjects are listed in the supplementary information (Table 1). In 14 subjects, there was no evidence of disease 
at the start of follow-up (i.e., the first CT or MRI scan of the head and neck region), five developed one or multiple paragangliomas (five HNPGL and one sPGL) and nine remained unaffected during a median follow-up time of 5.24 years (IQR: $3.26-6.54)$. In all other cases, follow-up started at diagnosis of the first PGL. Almost two-thirds of subjects $(n=145 ; 65 \%)$ presented with symptoms, and the median age at baseline was 39 years (range: 13-73) (Table 1). All but one subject visited the department of otorhinolaryngology, and biochemical screening was performed in $94 \%$.

\section{Development of new paragangliomas}

During a median follow-up time of 7 years (IQR: 4-12), 75 SDHD variant carriers (34\%) developed new head and neck paragangliomas and the number of subjects diagnosed with multiple HNPGL increased from 137 (62\%) to 171 (77\%). In addition, PCC or sPGL were detected in 21 subjects. Overall, $40 \%$ of all SDHD variant carriers developed new paragangliomas during follow-up. Carotid body tumors were encountered most frequently, followed by vagal body and jugulotympanic tumors (the distribution was approximately equal between males and females). In addition, HNPGL at other locations (e.g., proximal to the thyroid gland) were detected in five patients (Table 2).

The estimated fraction of SDHD variant carriers that developed at least one new head and neck paraganglioma ranged from 7\% (95\% CI: 3-10\%) after 2 years of followup, to $73 \%$ (95\% CI: $52-85 \%$ ) after 22 years, with a median time of 14.6 years (95\% CI: 11.5-16.1) (Fig. 2a). Males were more likely to develop new HNPGL compared to females (hazard ratio: 1.63, $p=0.01$ ), as were subjects that presented with symptoms compared to subjects that were asymptomatic at baseline (hazard ratio: $1.61, p=0.04$ ). The chance of developing new tumors decreased if more head and neck paragangliomas were already present (hazard ratio: $0.68, p<0.001$ ) (Fig. 2c, d and supplementary information, Table 2). There was no statistically significant effect of age.

\section{Clinical progression}

At the start of follow-up, 65\% of SDHD variant carriers were symptomatic. In addition, $24(31 \%)$ of previously asymptomatic subjects developed symptoms during followup, and $70(48 \%)$ already symptomatic patients reported new symptoms. In seven cases, the evolution of symptoms was unknown. In total, 94 (42\%) subjects reported new symptoms, including symptoms in 23 cases (24\%) attributable to tumors that developed during follow-up (the cumulative risk of developing a new symptomatic tumor is depicted in Fig. 2b). Furthermore, 70 (48\%) patients
Table 2 Number of subjects affected with paragangliomas and detected tumors at the start and end of follow-up (median follow-up time: 7 years)

\begin{tabular}{|c|c|c|}
\hline & $\begin{array}{l}\text { Start of } \\
\text { follow-up }\end{array}$ & $\begin{array}{l}\text { End of } \\
\text { follow-up }\end{array}$ \\
\hline Subjects & $n=222$ & $n=222$ \\
\hline No evidence of disease & $14(6 \%)$ & $9(4 \%)$ \\
\hline Affected with paragangliomas & $208(94 \%)$ & $213(96 \%)$ \\
\hline Head and neck paragangliomas & $207(93 \%)$ & $211(95 \%)$ \\
\hline Single head and neck paraganglioma & $70(32 \%)$ & $40(18 \%)$ \\
\hline $\begin{array}{l}\text { Multiple head and neck } \\
\text { paragangliomas }\end{array}$ & $137(62 \%)$ & $171(77 \%)$ \\
\hline $\begin{array}{l}\text { Pheochromocytoma and/or extra adrenal } \\
\text { sympathetic paraganglioma }\end{array}$ & $10(5 \%)$ & $31(15 \%)$ \\
\hline Tumors & $n=437$ & $n=570$ \\
\hline Head and neck paragangliomas & $424(97 \%)$ & $531(93 \%)$ \\
\hline Carotid body tumors & $271(62 \%)$ & $315(55 \%)$ \\
\hline Vagal body tumors & $100(23 \%)$ & $147(26 \%)$ \\
\hline Jugulotympanic tumors & $51(12 \%)$ & $64(11 \%)^{b}$ \\
\hline $\begin{array}{l}\text { Head and neck paragangliomas at } \\
\text { other locations }\end{array}$ & $2(0.5 \%)$ & $5(1 \%)$ \\
\hline Pheochromocytomas & $4(1 \%)$ & $16(3 \%)$ \\
\hline $\begin{array}{l}\text { Extra-adrenal sympathetic } \\
\text { paragangliomas }\end{array}$ & $9(2 \%)$ & $23(4 \%)$ \\
\hline
\end{tabular}

${ }^{\text {a }}$ Two hundred eight subjects underwent biochemical screening. At the end of follow-up, eight patients were diagnosed with multiple pheochromocytomas/extra-adrenal sympathetic paragangliomas

${ }^{\mathrm{b}}$ Twenty-three jugular paragangliomas and 12 tympanic paragangliomas. In the remaining 29 cases, no distinction could be made (i.e., jugulotympanic paragangliomas)

experienced progression of preexisting symptoms, of whom 37 also reported new symptoms. Both the exact date of diagnosis and the duration of symptoms were known for $79 \%$ of symptomatic patients. The mean age at onset was 36 $( \pm 13$; range: $9-72)$ years, and there was no statistically significant difference between males and females $(p=$ 0.73). In the remaining patients, symptoms had often been present for years, suggesting that the actual age at onset was lower. Jugulotympanic tumors were most often symptomatic, with hearing loss and tinnitus being the most commonly reported symptoms. Carotid and vagal body tumors most often caused a neck swelling, and to a lesser extent, pain, sensitivity, or a pressure sensation (Table 3).

In total, 211 SDHD variant carriers were diagnosed with head and neck paragangliomas. Twenty-three $(11 \%)$ subjects presented with cranial nerve dysfunction, of whom three developed additional cranial nerve palsies during follow-up. In total, 24 (11\%) patients developed new cranial nerve paresis or paralysis during follow-up, $21 \%$ of which was attributable to new head and neck paragangliomas (Table 4). 

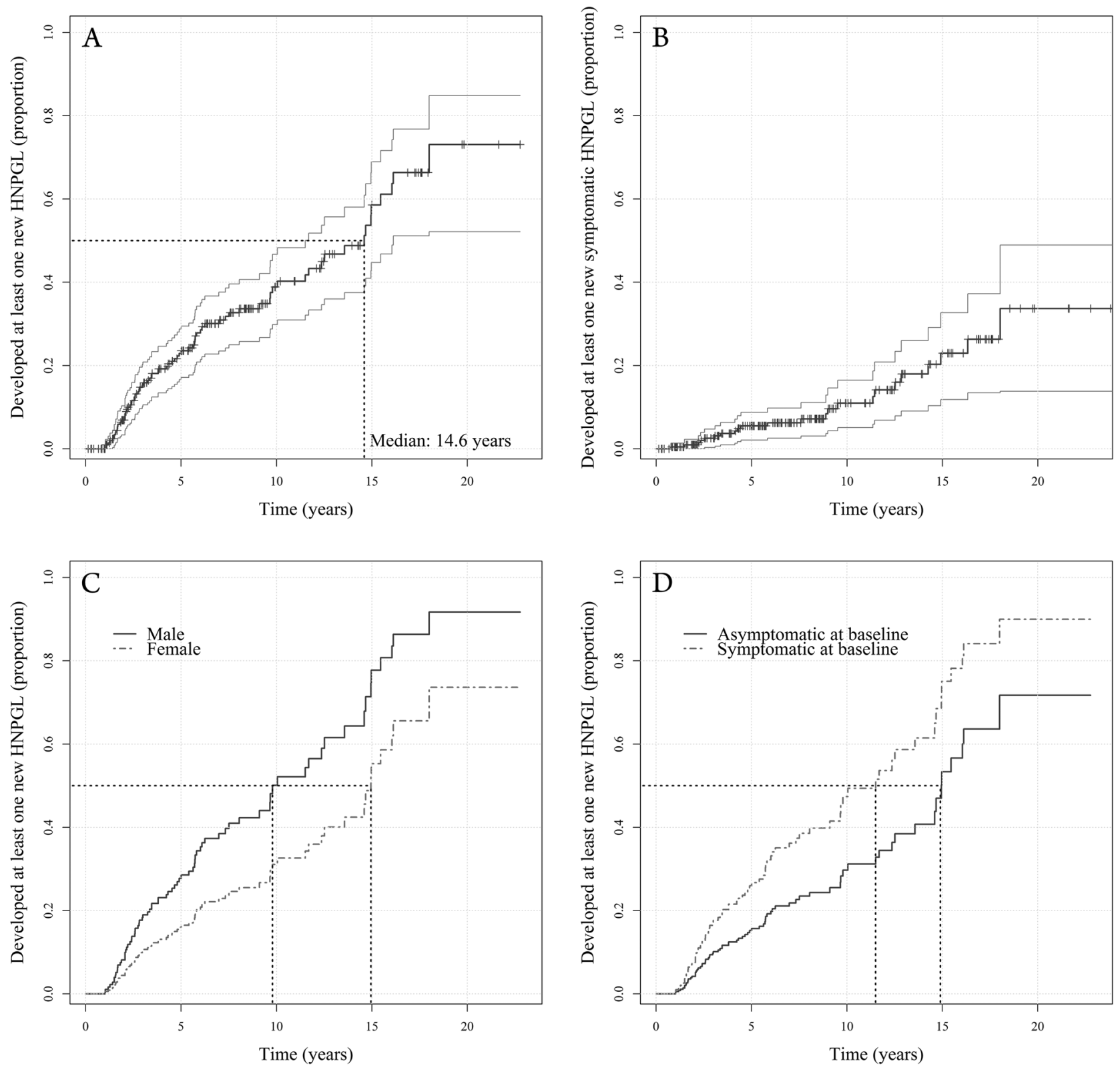

Fig. 2 The cumulative proportion of subjects that developed at least one new (symptomatic or asymptomatic) head and neck paraganglioma over time (a) and the cumulative proportion of subjects that developed at least one new symptomatic head and neck paraganglioma

Females more often reported new symptoms compared to males (odds ratio: 1.92, $p=0.03$ ), as did younger compared to older subjects (odds ratio per 10 years age increase: 0.76, $p=0.02$ ) (Supplementary information, Table 3). Both the number of HNPGL present at baseline and the number of new tumors were statistically significant predictors for the development of new symptoms (odds ratio of 1.53 and 1.90 , with $p$ values of 0.01 and 0.003 ). The former was the only significant predictor for the development of new cranial nerve paresis or paralysis (odds ratio of $1.69, p=0.03$ ).

(b), with 95\% confidence interval. c The risk of males versus females and (d) symptomatic versus asymptomatic patients, represented both for median or mean values of other predictors. The black dotted lines illustrate the estimated median time to the detection of a new tumor

Urinary catecholamine and/or $O$-methylated metabolites levels were elevated in $79(40 \%)$ patients at some point during follow-up, and one or multiple pheochromocytoma or extra-adrenal sympathetic paragangliomas were detected in 30 patients. In the remaining cases, increased urinary excretion rates of catecholamines and/or $O$-methylated metabolites were attributable to head and neck paragangliomas. In addition, in nine cases, catecholamine excess persisted or recurred after treatment for a PCC or sPGL, and could be attributed to the presence of HNPGL. In four other cases, both HNPGL and SPGL were 
Table 3 Symptoms present at diagnosis for both patients (211 SDHD variant carriers who were diagnosed with at least one head and neck paraganglioma and 2 with only an extra-adrenal sympathetic paraganglioma) and tumor locations separately

\begin{tabular}{|c|c|c|c|c|}
\hline & Patients & $\begin{array}{l}\text { Carotid body } \\
\text { tumors }(\%)\end{array}$ & $\begin{array}{l}\text { Vagal body } \\
\text { tumors }(\%)\end{array}$ & $\begin{array}{l}\text { Jugulotympanic tumors } \\
(\%)\end{array}$ \\
\hline & $n=213$ & $n=315$ & $n=147$ & $n=64$ \\
\hline Asymptomatic at diagnosis ${ }^{\mathrm{a}}$ & $67(31 \%)$ & $191(61 \%)$ & $103(70 \%)$ & $13(20 \%)$ \\
\hline \multirow[t]{2}{*}{ Symptomatic at diagnosis ${ }^{a}$} & $146(69 \%)$ & $112(36 \%)$ & $43(29 \%)$ & $48(75 \%)$ \\
\hline & $n=140^{\mathbf{a}}$ & $n=112$ & $n=43$ & $n=48$ \\
\hline Neck swelling ${ }^{\mathrm{b}}$ & $94(67 \%)$ & $103(92 \%)$ & $23(53 \%)$ & $0(0 \%)$ \\
\hline Pain/sensitivity/pressure & $24(17 \%)$ & $15(13 \%)$ & $5(12 \%)$ & $7(15 \%)$ \\
\hline Dysphagia & $13(9 \%)$ & $7(6 \%)$ & $10(23 \%)$ & $5(10 \%)$ \\
\hline Tinnitus (pulsatile) & $35(25 \%)$ & $2(2 \%)$ & $3(7 \%)$ & $33(69 \%)$ \\
\hline Tinnitus (non-pulsatile) & $4(3 \%)$ & $0(0 \%)$ & $0(0 \%)$ & $4(8 \%)$ \\
\hline Hearing loss & $30(21 \%)$ & $0(0 \%)$ & $0(0 \%)$ & $33^{\mathrm{c}}(69 \%)$ \\
\hline Dizziness & $6(4 \%)$ & $4(4 \%)$ & $2(5 \%)$ & $4(8 \%)$ \\
\hline $\begin{array}{l}\text { Obstructive sleep apnea } \\
\text { syndrome }\end{array}$ & $2(1 \%)$ & $4(4 \%)$ & $0(0 \%)$ & $0(0 \%)$ \\
\hline Dysphonia & $15(11 \%)$ & $5(4 \%)$ & $13(30 \%)$ & $8(17 \%)$ \\
\hline Other $^{\mathrm{d}}$ & $19(14 \%)$ & $9(8 \%)$ & $5(12 \%)$ & $12(25 \%)$ \\
\hline $\begin{array}{l}\text { Symptomatic catecholamine } \\
\text { excess }{ }^{\mathrm{e}}\end{array}$ & $8(6 \%)$ & $6(5 \%)$ & $3(7 \%)$ & $4(8 \%)$ \\
\hline
\end{tabular}

Occasionally, symptoms were attributable to a conglomerate of multiple tumors, or it could not be determined which tumor caused symptom(s) ${ }^{a}$ Discrepancies due to incomplete data

${ }^{\mathrm{b}}$ Bilateral in 17 subjects $(12 \%)$

${ }^{\mathrm{c}}$ Objectified in $85 \%$

${ }^{\mathrm{d}}$ For example, symptoms related to cranial nerve impairment (NVII, NIX, and NXII), syncope, coughing, dyspnea, globus sensation, (bloody) otorrhea, recurrent otitis, and fatigue

${ }^{\mathrm{e}}$ Attributable to catecholamine secreting head and neck paragangliomas in three subjects, and to pheochromocytomas or extra-adrenal sympathetic paragangliomas in five subjects

biochemically active. A biochemically silent extra-adrenal sympathetic paraganglioma was detected in one patient.

Eight (4\%) patients were diagnosed with metastatic paragangliomas. In four cases, this involved metastatic spread to locoregional lymph nodes, while in the remaining cases distant metastasis (pulmonary, skeletal, and/or hepatic metastasis) were present. Two patients died of metastatic disease, in a third case the cause of death was unknown and five patients are still in follow-up (mean follow-up time: 7.7 years). In four cases, the primary tumor was located in the head and neck region and in two cases the primary tumor was an sPGL. The remaining two patients were diagnosed with both HNPGL and sPGL and the location of the primary tumor was uncertain.

\section{Treatment}

A total of 108 patients were treated for 163 head and neck paragangliomas (112 carotid body, 18 vagal body, 13 jugulotympanic, 11 tympanic, 6 jugular paragangliomas, and 3 HNPGL at other locations). The majority of tumors were treated surgically (83\%), but radiation therapy $(15 \%)$, embolization (5\%), and octreotide (4\%) and lutetium (2\%) therapy were also used (as primary or adjuvant therapy). Treatment caused permanent cranial nerve injury in 24 patients $(22 \%)$, all but two cases of which were attributable to surgical treatment. The vagus nerve was most often affected, followed by the hypoglossal nerve (Supplementary information, Table 3). In addition, serious complications, including respiratory insufficiency and stroke, were reported in seven cases.

A total of 27 patients were treated for 16 PCC and 18 sPGL. Serious complications (such as pneumonia, radiation myelopathy and terminal kidney insufficiency) occurred in five cases.

\section{Discussion}

Lifelong follow-up is generally recommended in cases of hereditary paragangliomas. However, the risk of developing metachronous lesions has never been studied, nor are there any sizable studies reporting the evolution of symptoms or cranial nerve damage. In this study, we focused on SDHD 
Table 4 Cranial nerve paresis or paralysis for both patients (211 SDHD variant carriers diagnosed with head and neck paragangliomas, and 44 patients who presented with or developed cranial nerve dysfunction) and individual tumors

\begin{tabular}{|c|c|c|c|c|c|c|c|}
\hline & \multirow{2}{*}{$\begin{array}{l}\text { Patients (\% } \\
\text { of 211) }\end{array}$} & \multicolumn{2}{|c|}{ Carotid body tumors (\% of 315 ) } & \multicolumn{2}{|c|}{ Vagal body tumors (\% of 147 ) } & \multicolumn{2}{|c|}{ Jugulotympanic tumors (\% of 64$)$} \\
\hline & & $\begin{array}{l}\text { Present at } \\
\text { diagnosis }\end{array}$ & $\begin{array}{l}\text { Developed } \\
\text { during follow-up }\end{array}$ & $\begin{array}{l}\text { Present at } \\
\text { diagnosis }\end{array}$ & $\begin{array}{l}\text { Developed } \\
\text { during follow-up }\end{array}$ & $\begin{array}{l}\text { Present at } \\
\text { diagnosis }\end{array}$ & $\begin{array}{l}\text { Developed } \\
\text { during follow-up }\end{array}$ \\
\hline Facial nerve & $9(4 \%)$ & $0(0 \%)$ & $0(0 \%)$ & $0(0 \%)$ & $0(0 \%)$ & $4(6 \%)$ & $5(8 \%)$ \\
\hline Glossopharyngeal nerve & $5(2 \%)$ & $1(0.3 \%)$ & $0(0 \%)$ & $0(0 \%)$ & $1(1 \%)$ & $2(3 \%)$ & $1(2 \%)$ \\
\hline Vagal nerve & $30(14 \%)$ & $3(1 \%)$ & $5(2 \%)$ & $15(10 \%)$ & $6(4 \%)$ & $9(14 \%)$ & $4(6 \%)$ \\
\hline Accessory nerve & $6(3 \%)$ & $1(0.3 \%)$ & $0(0 \%)$ & $1(1 \%)$ & $0(0 \%)$ & $2(3 \%)$ & $3(5 \%)$ \\
\hline Hypoglossal nerve & $18(9 \%)$ & $4(1 \%)$ & $0(0 \%)$ & $5(3 \%)$ & $6(4 \%)$ & $8(13 \%)$ & $2(3 \%)$ \\
\hline Other & $3(1 \%)$ & $0(0 \%)$ & $0(0 \%)$ & $0(0 \%)$ & $0(0 \%)$ & $1(2 \%)$ & $2(3 \%)$ \\
\hline $\begin{array}{l}\text { Cranial nerve } \\
\text { dysfunction }\end{array}$ & $44(21 \%)$ & $5(2 \%)$ & $5(2 \%)$ & $19(13 \%)$ & $10(7 \%)$ & $15(23 \%)$ & $11(17 \%)$ \\
\hline
\end{tabular}

This table displays paresis or paralysis attributable to tumor progression, cranial nerve damage related to treatment is listed in the supplementary information (Table 4)

Note 1: There were no patients with bilateral vocal cord paralysis

Note 2: In two patients, a deficit of the vagus nerve and in an additional patient a deficit of the hypoglossal nerve could have been caused by treatment

Note 3: In one patient, it was unknown if deficits of cranial nerves NIX, NX, and NXII (attributable to a conglomerate of a vagal and jugulotympanic tumor) developed during follow-up

Note 4: In 16 cases, it was not certain which tumor caused cranial nerve dysfunction, and in 8 cases cranial nerve dysfunction was attributable to a conglomerate of multiple tumors

variant carriers. SDHD variants are predominantly associated with head and neck paragangliomas, with an estimated penetrance at age 70 ranging from $\sim 85$ to $100 \%$. Pheochromocytomas and extra-adrenal sympathetic paragangliomas are observed less frequently [8, 11, 20, 23-26]. This distinction was also apparent from our results, with 95\% of subjects diagnosed with HNPGL, compared to $15 \%$ with a PCC and/or sPGL.

On average, SDHD variant carriers face an $\sim 75 \%(95 \%$ CI: 52-85\%) risk for a new HNPGL over a follow-up period of 22 years. This risk is dependent on the number of tumors already present at first presentation. A surprising finding was that men were more prone to develop new head and neck paragangliomas compared to women. We previously found no statistically significant relation between sex and growth of HNPGL [27]. An increased growth rate of central nervous system hemangioblastomas, as well as an increased tumor burden, has been reported in male compared to female patients with von Hippel-Lindau disease (VHL). The authors suggested that this might be due to male hormonal influences [28]. Considering that PGLs are also a manifestation of VHL disease, that both VHL and SDHD tumors exhibit stabilization of HIF- $1 \alpha$, and that testosterone has been found to induce HIF-1 $\alpha$ function in rats, we postulate that male hormones may affect the development of paragangliomas [28-31]. However, further research will be required to establish whether testosterone is involved in tumorigenesis of SDHD-related paragangliomas and to confirm sex-related differences in SDHD-related disease.
Patients symptomatic at the start of follow-up were more likely to develop new HNPGL, indicating that asymptomatic SDHD variant carriers, i.e., screening-detected patients, may have a more favorable natural course. Furthermore, it should be noted that although recurrent event analysis revealed no significant relation between age and the development of new tumors, the hazard ratio depicts a risk ratio per time unit. Thus, subjects with a higher life expectancy do have a higher cumulative risk of developing new paragangliomas.

The relatively high percentage of SDHD variant carriers diagnosed with HNPGLs in this cohort, in comparison to previous reports, is attributable to our surveillance regimen and inclusion criteria $[11,13,20,26]$. In agreement with previous studies, carotid body tumors were most common, followed by vagal and jugulotympanic tumors. Due to their anatomic location, jugulotympanic tumors most frequently presented with symptoms [32, 33].

At the start of follow-up, $65 \%$ of subjects were symptomatic. During a median follow-up time of 8 years, $42 \%$, including previously asymptomatic SDHD variant carriers, reported new symptoms, $24 \%$ of which were attributable to new tumors. Not surprisingly, both the number of tumors present at the start of follow-up, as well as the number of new tumors were statistically significant predictors for the development of new symptoms. In line with an earlier observation of decreasing growth rates of carotid and vagal body paragangliomas with increasing age, there was a negative correlation between age and the development of new symptoms 
[27]. In addition, females reported new symptoms more often, independent of number of PGL and age. It is well established that there are gender-related differences in reporting physical symptoms $[34,35]$. Whether the underlying cause is primarily related to biological differences, bodily vigilance, recall bias, or social standards is unclear, but in population samples and in samples of medical patients women report symptoms more frequently [34, 35].

Twenty-three subjects (11\%) presented with cranial nerve dysfunction and 24 subjects (11\%) developed new cranial nerve deficits during follow-up. In most cases, dysfunction, primarily involving the vagus and hypoglossal nerve, was attributable to jugulotympanic and/or vagal body paragangliomas. It is noticeable that $21 \%$ of cranial nerve dysfunction was attributable to newly developed tumors. Treatment (mainly surgery) resulted in permanent cranial nerve dysfunction in $22 \%$ of treated patients. In particular, carotid and vagal body PGL treatment more often resulted in cranial nerve deficits compared to a "wait and scan" strategy. It should be noted that evaluation of treatment was not the main objective of this study and we have not reported results for different treatment modalities separately. However, we know from previous research that the risk of postoperative cranial nerve impairment is high, and almost inevitable if vagal body tumors are resected [36, 37]. The risk of cranial nerve damage following radiotherapy is considerably lower $(0-7.4 \%)$, although acute and late side effects should be taken into account and weighed against the generally favorable natural course of these tumors [36, 37].

It is generally thought that head and neck paragangliomas seldom release catecholamines. However, if biochemical screening includes the measurement of urinary 3-methoxytyramine, catecholamine excess is observed in $\sim 30 \%$ of patients with exclusively HNPGL [38]. Finally, the observed malignancy rate was comparable to the rate reported in literature $[11,39]$.

As imaging of the thorax and abdomen was only performed if there was evidence for increased catecholamine secretion or in case of suspect signs or symptoms, biochemically silent pheochromocytomas and extra-adrenal sympathetic paragangliomas may have gone undetected. In addition, the sensitivity and specificity of newer functional imaging techniques such as ${ }^{18} \mathrm{~F}$-fluordopa and ${ }^{68} \mathrm{Ga}-$ DOTATATE PET/CT have proven superior compared to ${ }^{123}$ I MIBG scintigraphy [40, 41]. An increased risk of pheochromocytoma has been associated with certain SDHD variants [11]. Although there is only limited evidence for other genotype-phenotype correlations, the results presented here may not be applicable to carriers of all SDHD variants [12]. Lastly, due to the retrospective nature of this study, symptoms may have been underreported [12].

As SDHD variants are associated with multifocal disease, lifelong follow-up is generally advised. In addition to confirming the high prevalence of multifocal disease, importantly we also showed that SDHD variant carriers face a substantial risk for new head and neck paragangliomas during follow-up ( $\sim 75 \%$ after 22 years). In addition, we detailed the clinical characteristics of 222 SDHD variant carriers, presenting the evolution of symptoms and cranial nerve dysfunction. While up to $50 \%$ of SDHD variant carriers reported new symptoms during a median of 8 years, new cranial nerve dysfunction was observed in only $11 \%$, less than in previous surgical series. The risks reported here should be taken into account when considering treatment strategies and counseling.

\section{Compliance with ethical standards}

Conflict of interest The authors declare that they have no conflict of interest.

\section{References}

1. Hensen EF, van Duinen N, Jansen JC, et al. High prevalence of founder mutations of the succinate dehydrogenase genes in the Netherlands. Clin Genet. 2012;81:284-8.

2. Taschner PEM, Jansen JC, Baysal BE, et al. Nearly all hereditary paragangliomas in the Netherlands are caused by two founder mutations in the SDHD gene. Genes Chromosom Cancer. 2001;31:274-81.

3. Taïeb D, Kaliski A, Boedeker CC, et al. Current approaches and recent developments in the management of head and neck paragangliomas. Endocr Rev. 2014;35:er20141026.

4. Hensen EF, Jordanova ES, van Minderhout IJHM, et al. Somatic loss of maternal chromosome 11 causes parent-oforigin-dependent inheritance in SDHD-linked paraganglioma and phaeochromocytoma families. Oncogene. 2004;23:4076-83.

5. Bayley J-P, Oldenburg RA, Nuk J, et al. Paraganglioma and pheochromocytoma upon maternal transmission of SDHD mutations. BMC Med Genet. 2014;15:111.

6. Hoekstra AS, Devilee P, Bayley J-P. Models of parent-of-origin tumorigenesis in hereditary paraganglioma. Semin Cell Dev Biol. 2015;43:1-8.

7. Hoekstra AS, Addie RD, Ras C, et al. Parent-of-origin tumorigenesis is mediated by an essential imprinted modifier in SDHD -linked paragangliomas: SLC22A18 and CDKN1C are candidate tumor modifiers. Hum Mol Genet. 2016;25:ddw218.

8. Neumann HPH, Pawlu C, Peczkowska M, et al. Distinct clinical features of paraganglioma syndromes associated with SDHB and SDHD gene mutations. JAMA. 2004;292:943-51.

9. HJLM Timmers, Gimenez-Roqueplo AP, Mannelli M, Pacak K. Clinical aspects of SDHx-related pheochromocytoma and paraganglioma. Endocr Relat Cancer. 2009;16:391-400.

10. Piccini V, Rapizzi E, Bacca A, et al. Head and neck paragangliomas: genetic spectrum and clinical variability in 79 consecutive patients. Endocr Relat Cancer. 2012;19:149-55.

11. Ricketts CJ, Forman JR, Rattenberry E, et al. Tumor risks and genotype-phenotype-proteotype analysis in 358 patients with germline mutations in SDHB and SDHD. Hum Mutat. 2010;31:41-51.

12. Evenepoel L, Papathomas TG, Krol N, et al. Toward an improved definition of the genetic and tumor spectrum associated with SDH germ-line mutations. Genet Med. 2015;17:610-20. 
13. Heesterman BL, Bayley JP, Tops CM, et al. High prevalence of occult paragangliomas in asymptomatic carriers of SDHD and SDHB gene mutations. Eur J Hum Genet. 2013;21:469-70.

14. Lepoutre-Lussey C, Caramella C, Bidault F, et al. Screening in asymptomatic SDHx mutation carriers: added value of (18)F-FDG PET/CT at initial diagnosis and 1-year follow-up. Eur J Nucl Med Mol Imaging. 2015;42:868-76.

15. Mediouni A, Ammari S, Wassef M, et al. Malignant head/neck paragangliomas. Comparative Study. Eur Ann Otorhinolaryngol Head Neck Dis. 2014;131:159-66.

16. Fruhmann J, Geigl JB, Konstantiniuk P, Cohnert TU. Paraganglioma of the carotid body: treatment strategy and SDH-gene mutations. Eur J Vasc Endovasc Surg. 2013;45:431-6.

17. Neumann HPH, Bausch B, McWhinney SR, et al. Germ-line mutations in nonsyndromic pheochromocytoma. N Engl J Med. 2002;346:1459-66.

18. Havekes B, van Der Klaauw AA, Weiss MM, et al. Pheochromocytomas and extra-adrenal paragangliomas detected by screening in patients with SDHD-associated head-and-neck paragangliomas. Endocr Relat Cancer. 2009;16:527-36.

19. van Hulsteijn LT, Heesterman B, Jansen JC, et al. No evidence for increased mortality in SDHD variant carriers compared with the general population. Eur J Hum Genet. 2015;23:1713-6.

20. van Hulsteijn LT, den Dulk AC, Hes FJ, Bayley JP, Jansen JC, Corssmit EPM. No difference in phenotype of the main Dutch SDHD founder mutations. Clin Endocrinol. 2013;79:824-31.

21. Amorim LDAF, Cai J. Modelling recurrent events: a tutorial for analysis in epidemiology. Int J Epidemiol. 2015;44:324-33.

22. Therneau TM. A package for survival analysis in S. version 2.38. 2015. https://CRAN.R-project.org/package $=$ survival

23. Hensen EF, Jansen JC, Siemers MD, et al. The Dutch founder mutation SDHD.D92Y shows a reduced penetrance for the development of paragangliomas in a large multigenerational family. Eur J Hum Genet. 2010;18:62-66.

24. Benn DE, Gimenez-Roqueplo AP, Reilly JR, et al. Clinical presentation and penetrance of pheochromocytoma/paraganglioma syndromes. J Clin Endocrinol Metab. 2006;91:827-36.

25. Benn DE, Robinson BG, Clifton-Bligh RJ. 15 Years of paraganglioma: clinical manifestations of paraganglioma syndromes types 1-5. Endocr Relat Cancer. 2015;22:T91-103.

26. Schiavi $\mathrm{F}$, Demattè $\mathrm{S}$, Cecchini $\mathrm{ME}$, et al. The endemic paraganglioma syndrome type 1: origin, spread, and clinical expression. J Clin Endocrinol Metab. 2012;97:637-41.

27. Heesterman BL, de Pont LMH, Verbist BM, et al. Age and tumor volume predict growth of carotid and vagal body paragangliomas. J Neurol Surg B Skull Base. 2017;78:497-505. in press
28. Lonser RR, Butman Ja, Huntoon K, et al. Prospective natural history study of central nervous system hemangioblastomas in von Hippel-Lindau disease. J Neurosurg. 2014;120:1055-62.

29. Selak MA, Armour SM, MacKenzie ED, et al. Succinate links TCA cycle dysfunction to oncogenesis by inhibiting HIF- $\alpha$ prolyl hydroxylase. Cancer Cell. 2005;7:77-85.

30. Hussain I, Husain Q, Baredes S, Eloy JA, Jyung RW, Liu JK. Molecular genetics of paragangliomas of the skull base and head and neck region: implications for medical and surgical management. J Neurosurg. 2014;120:321-30.

31. Chen Y, Fu L, Han Y, et al. Testosterone replacement therapy promotes angiogenesis after acute myocardial infarction by enhancing expression of cytokines HIF-1a, SDF-1a and VEGF. Eur J Pharmacol. 2012;684:116-24.

32. Gimenez-Roqueplo AP, Caumont-Prim A, Houzard C, et al. Imaging work-up for screening of paraganglioma and pheochromocytoma in SDHx mutation carriers: a multicenter prospective study from the PGL.EVA investigators. J Clin Endocrinol Metab. 2013;98:4578-87.

33. Woolen S, Gemmete JJ. Paragangliomas of the head and neck. Neuroimaging Clin N Am. 2016;26:259-78.

34. Barsky AJ, Peekna HM, Borus JF. Somatic symptom reporting in women and men. J Gen Intern Med. 2001;16:266-75.

35. Gijsbers Van Wijk CMT, Kolk AM. Sex differences in physical symptoms: the contribution of symptom perception theory. Soc Sci Med. 1997;45:231-46.

36. Suárez C, Rodrigo JP, Bödeker $\mathrm{CC}$, et al. Jugular and vagal paragangliomas: systematic study of management with surgery and radiotherapy. Head Neck. 2013;35:1195-204.

37. Suárez C, Rodrigo JP, Mendenhall WM, et al. Carotid body paragangliomas: a systematic study on management with surgery and radiotherapy. Eur Arch Otorhinolaryngol. 2014;271:23-34.

38. van Duinen N, Steenvoorden D, Kema IP, et al. Increased urinary excretion of 3-methoxytyramine in patients with head and neck paragangliomas. J Clin Endocrinol Metab. 2010;95:209-14.

39. Burnichon N, Rohmer V, Amar L, et al. The succinate dehydrogenase genetic testing in a large prospective series of patients with paragangliomas. J Clin Endocrinol Metab. 2009;94:2817-27.

40. Martucci VL, Pacak K. Pheochromocytoma and paraganglioma: diagnosis, genetics, management, and treatment. Curr Probl Cancer. 2014;38:7-41. https://doi.org/10.1016/j.currproblcancer. 2014.01.001.

41. Archier A, Varoquaux A, Garrigue P, et al. Prospective comparison of $68 \mathrm{Ga}$-DOTATATE and 18F-FDOPA PET/CT in patients with various pheochromocytomas and paragangliomas with emphasis on sporadic cases. Eur J Nucl Med Mol Imaging. 2016;43:1248-57. 